\title{
The flare phenomenon: still learning after 35 years
}

\author{
Ignac Fogelman
}

Published online: 9 September 2010

(C) Springer-Verlag 2010

These are interesting and challenging times for the isotope bone scan with regard to the identification of metastatic disease, with mounting evidence that the addition of SPECT improves sensitivity and specificity, SPECT/CT is even better, ${ }^{18} \mathrm{~F}$-fluoride PET/CT better still. In addition there is FDG PET/CT with improved sensitivity for skeletal metastases in certain diseases such as lung cancer [1] and with its ability to identify early marrow involvement, with the lurking threat from whole-body MRI never far away. Nevertheless our conventional, beloved bone scan which has served us so well over the years continues to be widely used due to its well-known advantages of simplicity, relatively low cost, high sensitivity and the ease of evaluating the whole skeleton, and in truth because many clinicians are as yet less knowledgeable about the newer methodologies. However, even with this familiar friend it would appear that we are still learning.

The flare phenomenon on a bone scan was first recognised in the mid 1970s and more widely reported upon in the early 1980s with Rossleigh et al. in 1984 [2] the first to identify 'new' lesions developing during the flare response (presumably existing small volume disease which was beyond the resolution of the methodology used). The flare phenomenon has always fascinated as conceptually the fact that a worsening scan is good news is counterintuitive. The theory is that if a bone scan is performed early (time interval to be clarified as will be discussed later) following

I. Fogelman $(\bowtie)$

King's College London,

Department of Nuclear Medicine,

Guy's Hospital Borough Wing,

Great Maze Pond,

London SE1 9RT, UK

e-mail: ignac.fogelman@kcl.ac.uk the successful treatment of bone metastases one may witness the skeleton mounting an intense osteoblastic response (reflecting healing), which makes individual lesions appear 'worse' (more intense tracer uptake at that site) and additional lesions, not identified on the original study may be visualised with subsequent resolution of the flare by 6 months. To date, in routine practice, this was unlikely to cause problems as bone scans are not generally performed within a few months following change in treatment and the flare response is known about and most nuclear medicine physicians will advise that there should be a significant interval between scans. However, this situation may well change due to the introduction of new, potent therapies and the need for earlier assessment in clinical trials.

Monitoring what happens to bone metastases is an important clinical issue in view of the high associated morbidity, but is notoriously difficult to achieve, and it is recognised that individual patients may show a mixed response to treatment, with for example improvement in soft tissue disease but with progression of bone metastases or with some bone lesions responding and others progressing. In recent years there have been major advances in oncology with a variety of new treatments, many of which have significant side effects and which are often extremely costly, and it is important to know as early as possible whether patients are responding. Further patients are often entered into clinical trials with fixed protocols, requiring new baseline bone scans and follow-up studies 'early' after instigation of treatment, and therefore optimising such protocols and indeed the correct interpretation of the studies becomes highly relevant.

In this volume Cook et al. [3] present interesting new data relating to the flare phenomenon in a prospective study of 99 newly diagnosed patients receiving hormonal treat- 
ment for prostate cancer. The authors' hypothesis was that a flare would amplify the signal and be specific for bone metastases. Were they successful? Absolutely yes and probably, as one cannot be $100 \%$ certain regarding specificity but this certainly appears likely to be the case. The four key points to note from this study being:

1. Repeat bone scans were at 6 weeks (flare usually assessed at 3 months) following instigation of treatment.

2. A higher percentage of patients with bone metastases showed a flare $(41 \%)$ than has previously been reported.

3. In those with bone lesions of uncertain aetiology a flare occurred in $20 \%$.

4. Bone metastases were identified in $11 \%$ of high-risk patients who were not thought to have skeletal involvement, i.e. with baseline scans that were negative.

It would appear from points 1 and 2 that the timing of a follow-up scan may be critical and the fact that a higher percentage of patients with bone metastases showed a flare than previously reported suggests that by scanning at 3 months one may miss the flare in some cases.

In those patients with equivocal bone lesions $20 \%$ showed a flare response, and while routine investigations would have clarified the nature of most, an incidental benefit of such a test may be in confirming the nature of problematic bone lesions as occasionally even with the most extensive and sophisticated imaging there are some lesions which remain equivocal.

This is the first time that patients thought to have no bone involvement on an isotope bone scan have been shown to have disease, early following successful treatment ('amplifying the signal') which is fascinating in itself but nevertheless the key clinical issues are:

1. Can these findings influence management?

2. Does a flare have prognostic significance?

With regard to the first point if one considers patients with prostate cancer in general (rather than individuals) this is somewhat unclear as it would appear that treatment decisions were correct in most patients, but for those in whom there was no evidence of bone involvement (thought to have local disease only) but with bone lesions identified by a flare response, this is important with staging changed from M0 to M1 and with potentially curative treatment now non-curative treatment and these patients are unlikely to benefit from radical radiotherapy. Additional work will be required to confirm these findings, their frequency and in clarifying cost-benefit issues before such a 'diagnostic' test could be routinely recommended. With regard to the second point it is not known whether a flare translates into patient survival but one would expect this to be the case. This issue is worthy of note and merits consideration for a well-designed prospec- tive study. A further question which cannot be answered at the present time but could be addressed in such a study is whether all patients with bone metastases who respond to treatment show a flare, i.e. is the flare phenomenon a universal finding with successful treatment?

It is important to consider that the results of the present study may not be representative of other malignancies which do not typically stimulate such an intense osteoblastic response in the skeleton as prostate cancer does. Thus the timing of the maximum intensity of a flare response may be disease dependent. There is biochemical evidence in breast cancer that the flare occurs early (certainly by 1 month) [4], but it is not known to what extent the bone scan lags behind this. This raises a further question as to whether treatment could be monitored more easily by biochemistry and while this merits further study it is probable that biochemical markers would miss cases with progressive disease, possibly those with small volume disease and those where 'new' metastases are identified for the first time with a follow-up scan.

It was initially stated that these are interesting and challenging times for the isotope bone scan and the data from Cook et al. [3] regarding the flare phenomenon provide a new potential application which requires further validation. It is worth pointing out that a flare may be easier to identify in a trial setting with quality control issues, such as image intensity corrected for and with careful comparison of studies, than in routine practice and if this test becomes established then it will be important to address the issue of subjective evaluation of scans and develop standardised approaches to quantifying tracer uptake by the skeleton.

Thirty-five years on and we are still discussing and learning about the flare phenomenon. A final thought relates to ${ }^{18} \mathrm{~F}$-fluoride, as I anticipate future reports of ${ }^{18} \mathrm{~F}$ fluoride flares and wonder what further surprises await us with what is surely the ultimate bone scan.

\section{References}

1. Krüger S, Buck AK, Mottaghy FM, Hasenkamp E, Pauls S, Schumann C, et al. Detection of bone metastases in patients with lung cancer: $99 \mathrm{mTc}-\mathrm{MDP}$ planar bone scintigraphy, $18 \mathrm{~F}$-fluoride PET or $18 \mathrm{~F}-\mathrm{FDG}$ PET/CT. Eur J Nucl Med Mol Imaging 2009;36:1807-12.

2. Rossleigh MA, Lovegrove FT, Reynolds PM, Byrne MJ, Whitney BP. The assessment of response to therapy of bone metastases in breast cancer. Aust N Z J Med 1984;14:19-22.

3. Cook GJR, Venkitaraman R, Sohaib AS, Lewington VJ, Chua SC, Huddart RA, et al. The diagnostic utility of the flare phenomenon on bone scintigraphy in staging prostate cancer. Eur J Nucl Med Mol Imaging 2010. doi:10.1007/s00259-010-1576-0.

4. Coleman RE, Mashiter G, Whitaker KB, Moss DW, Rubens RD, Fogelman I. Bone scan flare predicts successful systemic therapy for bone metastases. J Nucl Med 1988;29:1354-9. 